\title{
ON THE WEAKLY DAMPED HARMONIC OSCILLATOR*
}

\author{
By HOWARD R. BAUM (Aerodyne Research, Inc.)
}

Abstract. The initial-value problem for the differential equation

$$
u^{\prime \prime}(t)+\epsilon\left[a+\left(u^{\prime}\right)^{2}\right] u^{\prime}+u=0
$$

is studied under the assumption that $a$ and $\epsilon$ are positive constants and $\epsilon$ is small. Perturbation methods are used to obtain a first approximation to the solution that is uniformly valid in $t$. For any non-zero $a$ the solution ultimately decays exponentially with a time scale $(\epsilon a)^{-1}$. For sufficiently small $a$, however, the damping is dominated by the cubic damping term for times of order $\epsilon^{-1}$ and is thus algebraic in character. The frequency of the oscillations is reduced by an amount $\frac{1}{8}(\epsilon a)^{2}$ and is unaffected by the cubic damping to the order of approximation considered.

The initial-value problem for the equation

$$
u^{\prime \prime}(t)+\epsilon\left(u^{\prime}\right)^{3}+u+0 \quad \epsilon \ll 1
$$

is often used as an illustration of the multi-time scale perturbation technique [1], [2]. The first approximation to the solution for $u(0)=1, u^{\prime}(0)=0$ is well known and given by

$$
u=\left(1+\frac{3}{4} \tau\right)^{-1 / 2} \operatorname{cost}+O(\epsilon) ; \quad \tau=\epsilon t .
$$

The solution is so different in character from that associated with the linearly damped oscillator that it is instructive to consider the initial-value problem

$$
\begin{gathered}
u^{\prime \prime}(t)+\epsilon\left[a+\left(u^{\prime}\right)^{2}\right] u^{\prime}(t)+u(t)=0, \\
u(0)=1 ; \quad u^{\prime}(0)=\beta .
\end{gathered}
$$

This reduces to the cubic damped oscillator when $a=0$ and approaches the linear case as $a$ approaches infinity. The method of analysis is as described in the references. Write:

$$
\begin{gathered}
u \equiv u(s, \tau, \epsilon) ; \quad \tau=\epsilon t \\
t=s\left(1+\epsilon^{2} \lambda_{2}+\cdots\right), \quad \lambda_{2}=\text { constant. }
\end{gathered}
$$

Note that the variable $s$ is suggested by the fact that the solution to the linear problem shows a frequency shift of order $\epsilon^{2}$, while the variable $\tau$ characterizes the time scale on which the amplitude of the solution decays. The introduction of the extra variable is, of course, the key to the multi-time scale method. The problem in the new variables becomes:

$$
\begin{gathered}
\left(1-2 \epsilon^{2} \lambda_{2}+\cdots\right) u_{\Delta .}+2 \epsilon u_{s \tau}+\epsilon^{2} u_{\tau \tau}+u \\
+\epsilon\left[a+\left(u_{t}+\epsilon u_{\tau}\right)^{2}\right]\left(u_{\theta}+\epsilon u_{\tau}\right)+O\left(\epsilon^{3}\right)=0, \quad u(0,0, \epsilon)=1, \\
\left(1-\epsilon^{2} \lambda_{2}\right) u_{s}(0,0, \epsilon)+\epsilon u_{\tau}(0,0, \epsilon)+O\left(\epsilon^{3}\right)=\beta .
\end{gathered}
$$

* Received April 9, 1971. 
Now expand $u$ in a power series in $\epsilon$ as follows:

$$
u=u^{(0)}(\tau, s)+\epsilon u^{(1)}(\tau, s)+\epsilon^{2} u^{(2)}(\tau, s)+O\left(\epsilon^{3}\right)
$$

The equations and boundary conditions through $O\left(\epsilon^{2}\right)$ are given by

$$
\begin{gathered}
u_{s s}^{(0)}+u^{(0)}=0, \quad u_{s s}^{(1)}+u^{(1)}+2 u_{s \tau}^{(0)}+\left[a+\left(u_{s}^{(0)}\right)^{2}\right] u_{s}^{(0)}=0, \\
u_{s s}^{(2)}+u^{(2)}-2 \lambda_{2} u_{s s}^{(0)}+2 u_{s \tau}^{(1)}+u_{\tau \tau}^{(0)}+\left[a+3\left(u_{s}^{(0)}\right)^{2}\right]\left(u_{r}^{(0)}+u_{s}^{(1)}\right)=0, \\
u^{(0)}(0,0)=1 \quad u_{s}^{(0)}(0,0)=\beta, \\
u^{(1)}(0,0)=0 \quad u_{s}^{(1)}(0,0)+u_{\tau}^{(0)}(0,0)=0 .
\end{gathered}
$$

The zeroth-order equations and boundary conditions in (3) are satisfied by:

$$
u^{(0)}=A^{(0)}(\tau) \cos s+B^{(0)}(\tau) \sin s, \quad A^{(0)}(0)=1, \quad B^{(0)}(0)=\beta .
$$

Substituting this in the equations and boundary conditions for $u^{(1)}$ yields:

$$
\begin{aligned}
u_{s e}^{(1)}+u^{(1)}= & -\cos s\left\{2 B^{(0)}(\tau)+B^{(0)}\left[a+\frac{3}{4}\left(A^{(0) 2}+B^{(0) 2}\right]\right\}\right. \\
+ & \sin s\left\{2 A^{(0)}(\tau)+A^{(0)}\left[a+\frac{3}{4}\left(A^{(0) 2}+B^{(0) 2}\right)\right]\right\} \\
+ & \frac{B^{(0)}}{4}\left(3 A^{(0) 2}-B^{(0) 2}\right) \cos 3 s+\frac{A^{(0)}}{4}\left(3 B^{(0) 2}-A^{(0) 2}\right) \sin 3 s, \\
& u^{(1)}(0,0)=0, \quad u_{s}^{(1)}(0,0)=-A^{(0) \prime}(0) .
\end{aligned}
$$

In order that $u^{(1)}$ be uniformly as small as $u^{(0)}$ it is necessary that the coefficients of $\cos s$ and $\sin s$ in (4) be zero. This leads to the following systems for $A^{(0)}$ and $B^{(0)}$ :

$$
\begin{aligned}
& 2 B^{(0)^{\prime}}=-B^{(0)}\left[a+\frac{3}{4}\left(A^{(0) 2}+B^{(0) 2}\right)\right], \\
& 2 A^{(0))^{\prime}}=-A^{(0)}\left[a+\frac{3}{4}\left(A^{(0) 2}+B^{(0) 2}\right)\right], \\
& A^{(0)}(0)=1, \quad B^{(0)}(0)=\beta .
\end{aligned}
$$

The solution is easily obtained by considering the function $z \equiv A^{(0) 2}+B^{(0) 2}$. Adding the two equations together gives:

$$
d z / d \tau=-z\left(a+\frac{3}{4} z\right), \quad z(0)=1+\beta^{2} .
$$

Thus

$$
z=\frac{e^{-a r}}{1 /\left(1+\beta^{2}\right)+(3 / 4 a)\left(1-e^{-a r}\right)} .
$$

Now notice that both of Eqs. (5) are identical linear equations with initial conditions $\beta$ and one respectively. It then follows that $B^{(0)}=\beta A^{(0)}$. Hence:

$$
\begin{aligned}
z & =\left(1+\beta^{2}\right) A^{(0) 2}=\left(1+1 / \beta^{2}\right) B^{(0) 2}, \\
A^{(0)} & =e^{-a \tau / 2}\left\{1+\left(3\left(1+\beta^{2}\right) / 4 a\right)\left(1-e^{-a \tau}\right)\right\}^{-1 / 2}, \\
B^{(0)} & =\beta A^{(0)} .
\end{aligned}
$$

The above equations show clearly the roles played by the linear and cubic damping terms. For sufficiently large $a$ (but small $\epsilon a$ ) the solution approaches that of the linearly 
damped oscillator:

$u=e^{-a \tau / 2}\left\{\cos \left(1-(\epsilon a / 2)^{2}\right)^{1 / 2} t+(\beta+\epsilon a / 2)\left[1-(\epsilon a / 2)^{2}\right]^{-1 / 2} \sin \left(1-(\epsilon a / 2)^{2}\right)^{1 / 2} t\right\}$.

When $a$ is small the situation is more interesting. For times such that $\tau$ is $O(1), a \tau$ is small and $A^{(0)}$ is approximately $\left\{1+\frac{3}{4}\left(1+\beta^{2}\right) \tau\right\}^{-1 / 2}$. This is the first approximation to the cubic damped case. It does not represent the solution accurately when $\tau$ is $O\left(a^{-1}\right)$. The damping is thus initially controlled by the cubic term. However, when $a \tau$ is $O(1)$ $A^{(0)}$ is $O\left(a^{1 / 2}\right)$ in magnitude and the linear and cubic terms are approximately equal in size. As $\tau$ increases the linear damping becomes predominant and ultimately:

$$
A^{(0)} \sim e^{-a \tau / 2}\left\{4 a / 3\left(1+\beta^{2}\right)\right\}^{1 / 2} .
$$

The remaining task is to ensure that $u^{(1)}$ is uniformly small compared with $u^{(0)}$ for all times. Eqs. (5) ensure that this is true for times such that $\tau$ is $O(1)$. In order that this be true for all $\tau$ it is necessary to proceed further with the calculation of $u^{(1)}$. In so doing the necessity for a frequency shift will become apparent.

The solution of Eq. (4) is given by:

$$
\begin{aligned}
u^{(1)}=A^{(1)}(\tau) \cos s+B^{(1)}(\tau) \sin s+B^{(0)} / 32 & {\left[B^{(0) 2}-3 A^{(0) 2}\right] \cos 3 s } \\
& +A^{(0)} / 32\left[A^{(0) 2}-3 B^{(0) 2}\right] \sin 3 s .
\end{aligned}
$$

The coefficients of $\cos 3 s$ and $\sin 3 s$ decay like $A^{(0) 3}$ for large $\tau$. Thus, if $A^{(1)}$ and $B^{(1)}$ decay at least as rapidly as $A^{(0)}$, then $u^{(0)}$ approximates $u$ for all $t$.

$A^{(1)}$ and $B^{(1)}$ are determined by substituting the expressions for $u^{(0)}$ and $u^{(1)}$ into the third of Eqs. (3). Equating the coefficients of $\cos s$ and $\sin s$ to zero yields the following:

$$
\begin{aligned}
2 B^{(1) \prime} & +\left[a+\frac{3}{4}\left(3 B^{(0) 2}+A^{(0) 2}\right)\right]\left(B^{(1)}+A^{(0) \prime}\right)+2 \lambda_{2} A^{(0)}+A^{(0) \prime \prime} \\
& -\frac{3}{2} A^{(0)} B^{(0)} A^{(1)}+\frac{9}{128} A^{(0)}\left(A^{(0) 2}-3 B^{(0) 2}\right)\left(B^{(0) 2}-A^{(0) 2}\right) \\
& +\frac{3}{2} A^{(0)} B^{(0)}\left[B^{(0) \prime}+\frac{3}{32} B^{(0)}\left(B^{(0) 2}-3 A^{(0) 2}\right)\right]=0 \\
2 A^{(1) \prime} & +\left[a+\frac{3}{4}\left(3 A^{(0) 2}+B^{(0) 2}\right)\right]\left(A^{(1)}-B^{(0) \prime}\right)-2 \lambda_{2} B^{(0)}-B^{(0) \prime \prime} \\
& +\frac{3}{2} A^{(0)} B^{(0)} B^{(1)}+\frac{9}{128} B^{(0)}\left(B^{(0) 2}-3 A^{(0) 2}\right)\left(B^{(0) 2}-A^{(0) 2}\right) \\
& +\frac{3}{2} A^{(0)} B^{(0)}\left[A^{(0) \prime}-\frac{3}{3^{32}} A^{(0)}\left(A^{(0) 2}-3 B^{(0) 2}\right)\right]=0 .
\end{aligned}
$$

First consider the case when $a \neq 0$. When $a \tau$ is large, Eqs. (8) can be written as:

$$
\begin{gathered}
2 B^{(1) \prime}+\left[a+O\left(e^{-a \tau}\right)\right] B^{(1)}+O\left(e^{-a \tau}\right) A^{(1)}+\left(O\left(e^{-3 a \tau / 2}\right)\right)+a A^{(0) \prime}+2 \lambda_{2} A^{(0)}+A^{(0) \prime \prime}=0 ; \\
2 A^{(1) \prime}+\left[a+O\left(e^{-a \tau}\right)\right] A^{(1)}+O\left(e^{-a \tau}\right)+O\left(e^{-3 a \tau / 2}\right)-a B^{(0) \prime}-2 \lambda_{2} B^{(0)}-B^{(0) \prime \prime}=0
\end{gathered}
$$

The solutions to the homogeneous equations for large time are thus proportional to $\exp (-a \tau / 2)$. However, the explicitly displayed inhomogeneous terms in (9) are also proportional to exp $(-a \tau / 2)$ for large $\tau$ unless $-a^{2} / 2+2 \lambda_{2}+a^{2} / 4=0 ; \lambda_{2}=a^{2} / 8$. The variable $s$ is then given in terms of $t$ by

$$
s=t\left(1-\left((a \epsilon)^{2} / 8\right)+O\left(\epsilon^{3}\right)\right) .
$$

Note that this is exactly the amount by which the frequency is reduced in the linearly damped oscillator. This must be the case since the only terms in Eq. (9) involved in the determination of $\lambda_{2}$ arise from the linear terms in Eq. (1). The cubic damping term 
then plays no role in the frequency shift to this order in $\epsilon$ for $a=0$. Indeed, Eq. (10) predicts no shift at all for $a=0$. While this result will turn out to be true, the above analysis is not applicable when $a=0$ and a separate study is required.

When $a=0$, Eqs. (5) and the relation $B^{(0)}=\beta A^{(0)}$ can be used to reduce Eqs. (8) to

$$
\begin{gathered}
2 B^{(1)^{\prime}}+\frac{3}{4}\left(1+2 \beta^{2}\right) A^{(0) 2} B^{(1)}-\frac{3}{2} \beta A^{(0) 2} A^{(1)} \\
\quad=-2 \lambda_{2} A^{(0)}+\mathrm{r}^{9} \frac{9}{28}\left(\beta^{2}+1\right)\left(15 \beta^{2}-1\right) A^{(0) 5}, \\
2 A^{(1)^{\prime}}+\frac{3}{4}\left(3+\beta^{2}\right) A^{(0) 2} A^{(1)}+\frac{3}{2} \beta A^{(0) 2} B^{(1)}=2 \lambda_{2} \beta A^{(0)}+\frac{9}{128} \beta\left(1+\beta^{2}\right) 2 A^{(0) 5} .
\end{gathered}
$$

First consider the inhomogeneous solutions to Eq. (11). These consist of two terms. The terms independent of $\lambda_{2}$ contribute a particular solution of the form

$$
B^{(1)}=c A^{(0) 3}, \quad A^{(1)}=d A^{(0) 3} .
$$

Substitution into (11) gives:

$$
c=(3 / 64)\left(\beta^{2}+1\right)\left(1-7 \beta^{2}\right), \quad d=-(3 \beta / 8)\left(1+\beta^{2}\right) .
$$

These inhomogeneous solutions decay faster than $A^{(0)}$. Moreover, a non-zero $\lambda_{2}$ contributes inhomogeneous solutions proportional to $\left(A^{(0)}\right)^{-1 / 2}$. Such solutions actually grow with $\tau$, and it is clear that if $u^{(0)}$ is to be the uniformly valid first approximation, $\lambda_{2}$ must be zero.

Finally the homogeneous solutions are obtained as follows. Define a new independent variable $\theta$ by:

$$
d \theta / d \tau=\frac{3}{4} A^{(0) 2}, \quad \theta=1 /\left(1+\beta^{2}\right) \log \left[1+\frac{3}{4}\left(1+\beta^{2}\right) \tau\right] .
$$

The homogeneous version of (11) is then:

$$
\begin{aligned}
& 2\left(d B^{(1)} / d \theta\right)+\left(1+3 \beta^{2}\right) B^{(1)}-2 \beta A^{(1)}=0, \\
& 2\left(d A^{(1)} / d \theta\right)+\left(3+\beta^{2}\right) A^{(1)}+2 \beta B^{(1)}=0 .
\end{aligned}
$$

Solutions of the form $\exp (p \theta)$ give values

$$
\begin{array}{ll}
p=-\left(1+\beta^{2}\right) \pm \frac{1}{2}\left(1-6 \beta^{2}+\beta^{4}\right)^{1 / 2} ; & 1-6 \beta^{2}+\beta^{4}>0, \\
p=-\left(1+\beta^{2}\right) \pm \frac{1}{2}\left(\left|1-6 \beta^{2}+\beta^{4}\right|\right)^{1 / 2} ; & 1-6 \beta^{2}+\beta^{4}<0 .
\end{array}
$$

The homogeneous solutions are thus:

$$
\begin{gathered}
{\left[1+\frac{3}{4}\left(1+\beta^{2}\right) \tau\right]^{-1 \pm \omega / 2}, \quad \begin{array}{cr}
1-6 \beta^{2}+\beta^{4}>0, & \omega=\left(1-6 \beta^{2}+\beta^{4}\right)^{1 / 2} /\left(1+\beta^{2}\right) ; \\
{\left[1+\frac{3}{4}\left(1+\beta^{2}\right) \tau\right]^{-1}\left\{\cos (\Omega / 2) \log \left[1+\frac{3}{4}\left(1+\beta^{2}\right) \tau\right],\right.} & \left.\sin (\Omega / 2) \log \left[1+\frac{3}{4}\left(1+\beta^{2}\right) \tau\right]\right\}, \\
1-6 \beta^{2}+\beta^{4}<0, \\
\Omega=\left(6 \beta^{2}-1-\beta^{4}\right)^{1 / 2} /\left(1+\beta^{2}\right) .
\end{array}}
\end{gathered}
$$

Since $\omega \leq 1$ for all $\beta$, the homogeneous solutions always decay as fast as or faster than $A^{(0)}$. Thus, when $a=0$, the choice $\lambda_{2}=0$ will insure that $\epsilon u^{(1)}$ is uniformly smaller than $u^{(0)}$ for all $t$.

\section{REFERENCES}

[1] G. F. Carrier and C. E. Pearson, Ordinary differential equations, Blaisdell, Waltham, Mass., 1968, p. 210

[2] J. D. Cole, Perturbation methods in applied mathematics, Blaisdell, Waltham, Mass. 1968, p. 91 